\title{
From Teledermatology to Dermatology Artificial Intelligence: Will Teledermatology Exist in the Next 2 Years?
}

\author{
Alexander Börve ${ }^{1,2}$ \\ ${ }^{1}$ Sahlgrenska Academy, University of Gothenburg, Gothenburg, Sweden \\ ${ }^{2}$ iDoc24 Inc, Berkeley, CA, United States \\ Corresponding Author: \\ Alexander Börve \\ Sahlgrenska Academy \\ University of Gothenburg \\ Medicinaregatan 3 \\ Gothenburg, 41390 \\ Sweden \\ Phone: 46317860000 \\ Email: alexander@idoc24.com
}

\section{Abstract}

Background: Dermatology has been proven to be well suited for store-and-forward telemedicine triaging. With the reduced cost of computer power and readily available deep convolutional neural networks, using the digital images collected with store-and-forward, machine learning has made it possible to create artificial intelligence (AI) models. The AI models can analyze new digital images taken with a smartphone camera and return reliable dermatology outputs within seconds.

Objective: The aim of this study is to demonstrate the shift from teledermatology to dermatology AI.

Methods: A literature search was conducted and experience from a web-based teledermatology service was also considered.

Results: There has been a slow uptake of teledermatology in a clinical setting and by consumers. The development of AI dermatology models has gained momentum over the last few years. Studies have shown that AI dermatology is on par with teledermatology to deliver accurate diagnoses for the most common dermatology pathologies.

Conclusions: Teledermatology has still not gained mass adoption, both clinically and directly by consumers. The fast pace of AI dermatology development indicates that the technology will surpass store-and-forward teledermatology as a first point of digital consultation in dermatology.

Conflicts of Interest: $\mathrm{AB}$ is the owner of iDoc24 Inc.

(iproc 2022;8(1):e36907) doi: 10.2196/36907

\section{KEYWORDS}

artificial intelligence; teledermatology; dermatology

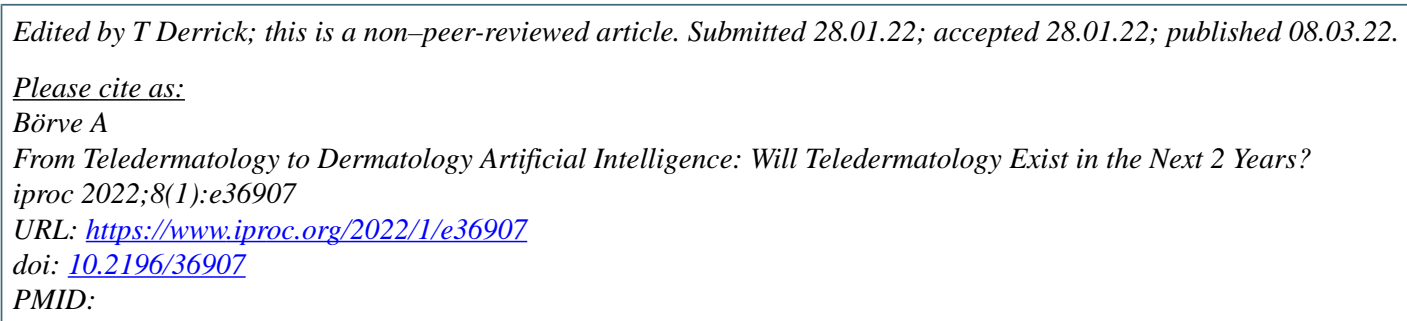


(C)Alexander Börve. Originally published in Iproceedings (https://www.iproc.org), 08.03.2022. This is an open-access article distributed under the terms of the Creative Commons Attribution License (https://creativecommons.org/licenses/by/4.0/), which permits unrestricted use, distribution, and reproduction in any medium, provided the original work, first published in Iproceedings, is properly cited. The complete bibliographic information, a link to the original publication on https://www.iproc.org/, as well as this copyright and license information must be included. 